\title{
Self Diagnostic Accelerometer Ground Testing on a C-17 Aircraft Engine
}

\author{
Roger P. Tokars \\ Glenn Research Center \\ Cleveland, OH 44135 \\ 216-433-8771 \\ Roger.P.Tokars@nasa.gov
}

\author{
John D. Lekki \\ Glenn Research Center \\ Cleveland, OH 44135 \\ 216-433-5650 \\ John.D.Lekki@nasa.gov
}

Abstract-The self diagnostic accelerometer (SDA) developed by the NASA Glenn Research Center was tested for the first time in an aircraft engine environment as part of the Vehicle Integrated Propulsion Research (VIPR) program. The VIPR program includes testing multiple critical flight sensor technologies. One such sensor, the accelerometer, measures vibrations to detect faults in the engine. In order to rely upon the accelerometer, the health of the accelerometer must be ensured. Sensor system malfunction is a significant contributor to propulsion in flight shutdowns (IFSD) which can lead to aircraft accidents when the issue is compounded with an inappropriate crew response. The development of the SDA is important for both reducing the IFSD rate, and hence reducing the rate at which this component failure type can put an aircraft in jeopardy, and also as a critical enabling technology for future automated malfunction diagnostic systems. The SDA is a sensor system designed to actively determine the accelerometer structural health and attachment condition, in addition to making vibration measurements. The SDA uses a signal conditioning unit that sends an electrical chirp to the accelerometer and recognizes changes in the response due to changes in the accelerometer health and attachment condition. In an effort toward demonstrating the SDA's flight worthiness and robustness, multiple SDAs were mounted and tested on a $\mathrm{C}-17$ aircraft engine. The engine test conditions varied from engine off, to idle, to maximum power. The two SDA attachment conditions used were fully tight and loose. The newly developed SDA health algorithm described herein uses cross correlation pattern recognition to discriminate a healthy from a faulty SDA. The VIPR test results demonstrate for the first time the robustness of the SDA in an engine environment characterized by high vibration levels.

\section{Table of Contents}

1. INTRODUCTION 1

2. THEORY 2

3. METHOD .3

4. RESULTS . 4

5. CONCLUSION 7

REFERENCES .8

U.S. Government work not protected by U.S. copyright

\section{INTRODUCTION}

$\triangle$ ccelerometers are commonly used for health

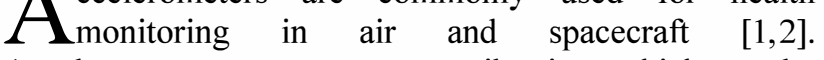
Accelerometers measure system vibrations which may be correlated with faults. In order for the vibrations to correctly couple into and be measured by the accelerometer, the accelerometer attachment condition and health must be ensured. A serious concern is relying upon a faulty accelerometer in a critical system that takes corrective action, such as an automated shut down of an engine [3], or where a faulty sensor will lead to inappropriate crew response. Propulsion System Malfunction plus Inappropriate Crew Response (PSM+ICR) is an issue that continues to be a significant contributor to aviation accidents worldwide [4]. The self diagnostic accelerometer (SDA) is directly applicable to this issue as it addresses sensor failure, which is one of the more prevalent component failures leading to in flight shutdowns and hence potentially contributing to PSM+ICR [5]. The SDA reduces the probability of using faulty accelerometer data by checking the health and attachment condition of the accelerometer in situ.

The capabilities of the SDA have been investigated previously. Testing of early designs of the SDA has shown a promising capability to diagnose sensor health and attachment condition [6,7]. Using the well known properties of piezoelectrics, the SDA applies to the accelerometer's piezoelectric crystal a low voltage, swept frequency electrical signal or chirp. The resultant electrical output from the accelerometer considered in the frequency domain contains a pattern of resonant antiresonant pairs that may be used to characterize the health and attachment condition of the accelerometer. An accelerometer electrical fault, such as an open or short circuit, is easily detectable because the diagnostic response trends to 1 . when the accelerometer is shorted and trends to zero with an open circuit. Damage to the sensor itself causes additional resonant antiresonant pairs which are also easily detectable because the diagnostic response pattern is changed significantly. An attachment fault causes smaller shifts and distortions to the pattern which makes that kind of fault more difficult to detect. For this reason the attachment fault was chosen as the primary focus of most recent work. Such recent efforts have included simulated engine tests with a shaker and oven at specific vibration and temperature levels [7]. Testing described here takes the SDA development a step 
further by installing SDAs in an operating turbojet engine installed on a grounded aircraft. For this work, the SDAs were installed on a $\mathrm{C}-17$ aircraft engine as part of the Vehicle Integrated Propulsion Research (VIPR) engine tests in 2011.

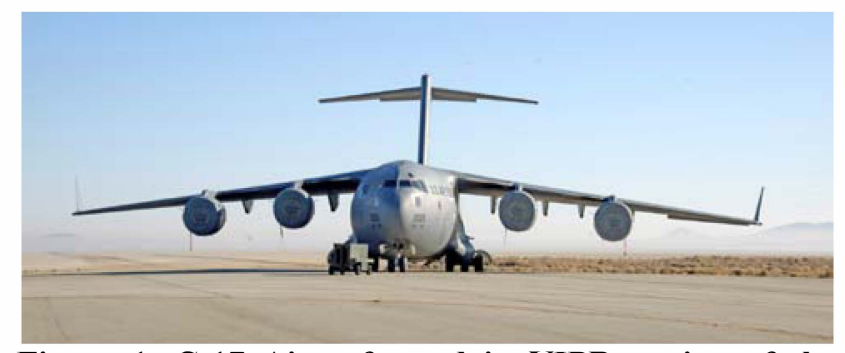

Figure 1. C-17 Aircraft used in VIPR testing of the SDA.

In addition to testing the SDA for the first time in the extreme environment of an operating jet engine, the newly developed cross correlation pattern recognition software was also tested to discriminate a faulty SDA from a healthy SDA.

SDA engine testing on the $\mathrm{C}-17$ included testing with the SDA installed at multiple engine locations, attachment conditions, and engine vibration levels. The two engine locations of interest for SDA installation were the BFlange and the Gearbox locations, which correspond to locations under the cowling described in a later section. Two SDAs were installed at each location. In each location, one accelerometer was tightly attached and the other loosely attached. These tight/loose attachment conditions were switched halfway through testing in order to demonstrate the SDA's ability to predict the attachment fault. The SDAs were exposed to engine vibrations that resulted from engine off, engine idle, and engine maximum thrust levels. Engine vibrations were also monitored with existing legacy accelerometers installed near the SDAs.

\section{THEORY}

The SDA system consists of a piezoelectric accelerometer and a signal analyzer. The piezoelectric accelerometer normally operates by generating a charge when it senses acceleration based on the direct piezoelectric effect as given in Eq. 1. The SDA system uses the converse piezoelectric effect as given in Eq. 2. The SDA signal analyzer applies a time varying voltage to the accelerometer's piezoelectric crystal which causes a corresponding strain in the crystal.

$$
\begin{aligned}
& P=d T \\
& S=d E
\end{aligned}
$$

where $P$ is the electric polarization, $d$ is the piezoelectric constant, $T$ is the stress, $S$ is the strain, and $E$ is the electric field [9].
By sending an electrical chirp to the accelerometer and measuring the response (output/input), as seen in Fig. 2 , the signal analyzer can monitor for any changes in the response. The changes in the response pattern depend on the mechanical and electrical impedance of the SDA system. The mechanical and electrical impedance is related to the health and attachment of the accelerometer.

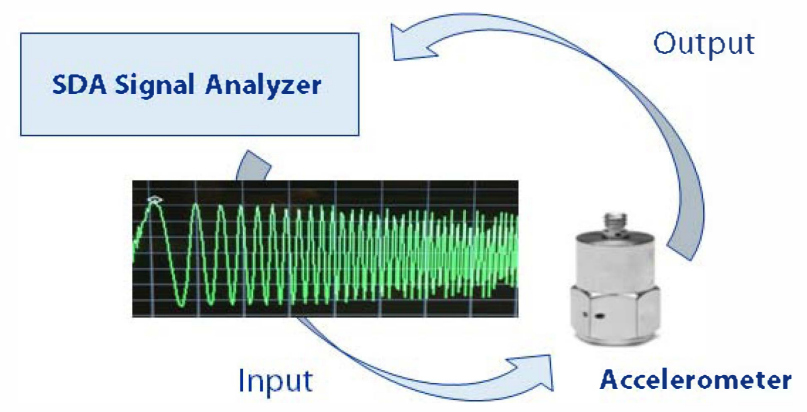

Figure 2. Model of the SDA system.

The SDA diagnostic response, measured in the frequency domain, is unique to each accelerometer and its base attachment condition. The diagnostic response takes the form of a series of resonant antiresonant pairs that shift and change according to the health and attachment condition of the accelerometer. A baseline reference signal is taken for each SDA in the tightly attached condition. Deviation from this reference signal may be used as an indicator of the health and attachment condition of the accelerometer. An example of the frequency response (output signal divided by input signal) of a healthy accelerometer is shown in Fig. 3. The resonant frequencies of the system stand out as spikes in the signal. The SDA diagnostic chirp ranges between 30 and $80 \mathrm{kHz}$. This is outside and thus doesn't interfere with the typical signal bandwidth $(<25$ $\mathrm{kHz})$ of the accelerometer used as a

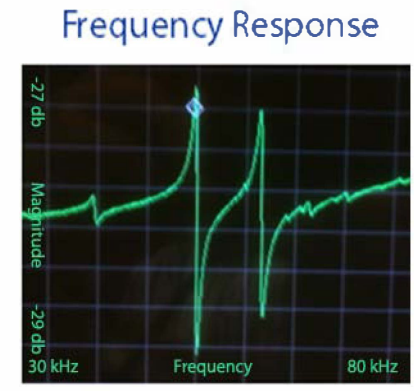

Figure 3. SDA frequency response example of a healthy sensor. vibration sensor.

Empirical models have shown a predictable change in the accelerometer diagnostic response caused by a change in the accelerometer's environment [7] as shown in Fig. 4. These changes include changes in temperature, mount torque, and structural integrity.

Temperature increase causes the frequency response pattern to shift up and to the left in the magnitude vs. frequency plot as seen Fig. 4a. The frequency response pattern generally remains the same with the number of resonances and the separation between them remaining unchanged. 
Mounting torque decrease (loosening the attachment condition) causes existing resonances to shift to the left and become smaller in the magnitude vs. frequency plot as seen in Fig. 4b. As the attachment condition transitions from tight to loose, the resonances disappear entirely and new resonances may spontaneously appear elsewhere in the diagnostic band. The number of resonances and the separation between them changes so much that the frequency response pattern for the tight and loose condition are completely different and can, as a result, be used to determine the sensor attachment condition.

Accelerometer structural damage results in a change in the frequency response pattern inconsistent with the reference healthy condition frequency response pattern. Typically a damaged accelerometer will have additional resonances. If the piezoelectric crystal is critically damaged, the electrical connection could be disconnected resulting in an entirely unresponsive accelerometer.

a)
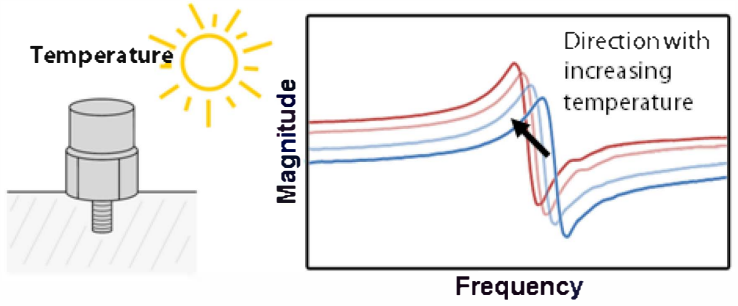

b)
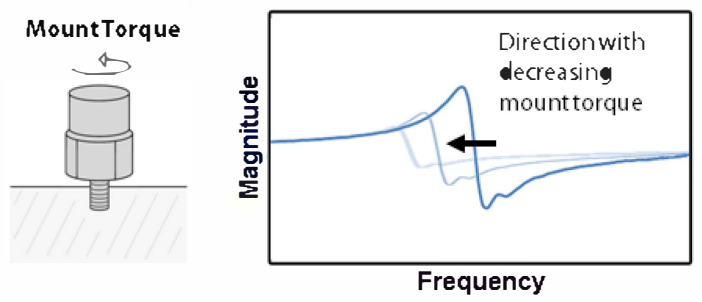

c)

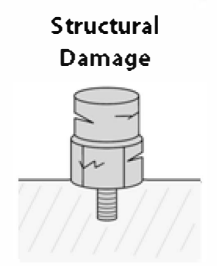

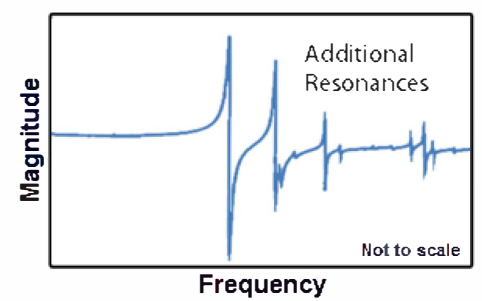

Figure 4. SDA Resonant frequency changes due to a) temperature, b) mount torque and c) structural damage.

Health algorithms are used to track the resonant frequencies in order to detect changes in the mechanical system. Previous tests of the SDA used a derivative analysis health algorithm [8] which tracked the resonant frequencies by taking the derivative of the SDA signal. The algorithm of choice for this paper uses cross correlation pattern recognition to detect changes. The advantage of cross correlation is that it takes into account the entire diagnostic response pattern instead of just tracking the resonant frequencies as before.
In the SDA cross correlation method we determine the correlation between the reference healthy SDA frequency response and an experimental SDA frequency response. A high correlation suggests that the experimental condition is healthy. A low correlation suggests otherwise. When low correlation suggests otherwise, a reference fault condition can be cross correlated with the experimental condition to confirm the type of fault condition.

The SDA cross correlation method utilizes Eq. 3 where refr is the reference dataset, expr is the experiment dataset, $m$ is the frequency, $n$ is a frequency shift, and the subscript $A v g$ denotes the average of the dataset. The SDA cross correlation requires both the datasets should be from a relevant frequency range. In this work the reference and experimental datasets are limited to the range from $30 \mathrm{kHz}$ to $80 \mathrm{kHz}$. Outside this range the points are ignored by setting them to zero. A high correlation is indicated when $r[n]$ is close to 1 . A low correlation is indicated when $r[n]$ is close to 0 .

$$
r[n]=\frac{\sum_{m=30 \mathrm{kHz}}^{80 \mathrm{kHz}}\left\{\begin{array}{c}
\left(\operatorname{refr}[\mathrm{m}]-\operatorname{refr}_{A v g}\right) \cdot \\
\left(\operatorname{expr}[n+m]-\operatorname{expr}_{A v g}\right)
\end{array}\right\}}{\sqrt{\sum_{m=30 \mathrm{kHz}}^{80 \mathrm{kHz}}\left\{\left(\operatorname{refr}[m]-\operatorname{refr}_{A v g}\right)^{2}\right\}}} .
$$

The maximum value of $r[n]$ in equation 3 is used to determine the Health Index. The maximum of the normalized cross correlation function is evaluated over the range $-1500 \mathrm{~Hz}<=n<=1500 \mathrm{~Hz}$. The $3 \mathrm{kHz}$ range is the maximum expected resonant frequency shift from both temperature and torque as determined in the laboratory and seen in Figs. 4a and 4b. The maximum frequency shift for the tightness extremes of 10 in-lbs to 40 in-lbs yielded approximately $500 \mathrm{~Hz}$ shift. The maximum frequency shift for $150^{\circ} \mathrm{C}$ temperature change yielded approximately $1600 \mathrm{~Hz}$ shift. The sum of these two numbers and then rounded up to the nearest thousand resulted in the $3 \mathrm{kHz}$ range.

$$
\text { Health Index }=\text { maximum }(r[n])
$$

\section{Method}

Strict operating procedures were followed during testing of the SDA system on the C-17 engine in order to obtain accurate and consistent data as test parameters were varied. The SDA system was tested at engine off, engine idle, and engine maximum thrust for a minimum of 2 minutes for each condition in order to collect an adequate amount of data from all sensors. Continuous SDA data was collected and time synchronized with the aircraft sensor data and existing legacy accelerometers. The legacy accelerometers were located next to the SDAs in order to provide reference vibration measurements. 
Four SDAs were installed on the engine under the cowling at two locations: the B-Flange and Gearbox as indicated in Fig. 5. Each location had one SDA tightened

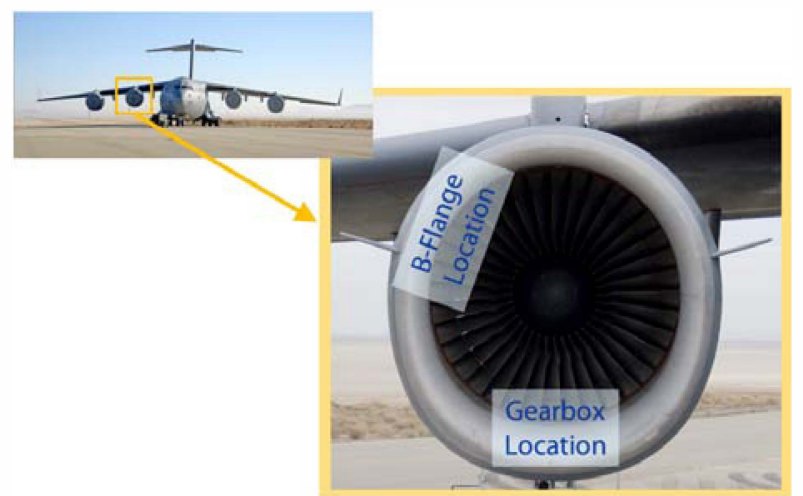

Figure 5. SDA installations located inside the $\mathrm{C}-17$ engine at the B-Flange and Gearbox.

at 30 in-lbs of torque and the other SDA loosened a quarter turn loose from the tight 30 in-lbs torque. The SDAs tight/loose configurations were switched halfway through testing in order to compare the difference between the two conditions from the same accelerometer As a safety precaution, safety wire, seen in Fig. 6, secured the accelerometer to the engine mount to reduce the chances of the accelerometer falling off and damaging the engine. The safety wire also served the purpose of keeping the accelerometer tight/loose condition consistent. Laboratory testing of the reference tight and loose conditions with and without the safety wire confirmed that the safety wire did not significantly alter the resonances measured.

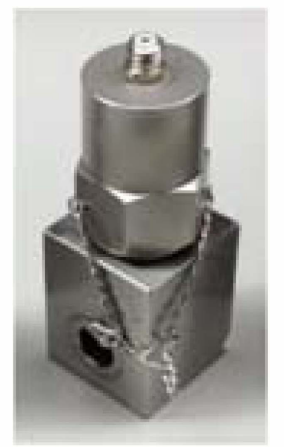

Figure 6. Accelerometer secured to the engine mount with safety wire.
A schematic of the installation of the SDA system on the $\mathrm{C}-17$ aircraft is shown in Fig. 7. The four accelerometers, labeled as SDA \#0, \#1, \#3 and \#4, were installed on the engine. SDAs $\# 0$ and \#1 were installed in the B-Flange location. SDAs \#3 and \#4 were installed in the Gearbox location. The accelerometers were connected to $150 \mathrm{ft}$ cables. The cables passed through an opening in the engine framing, traced along the aircraft wing, and then entered the fuselage/cargo bay where they connected into a switch box. The switch box was used to select which accelerometer was connected to the signal analyzer. The computer controlled the signal analyzer and saved the resulting diagnostic data with the aircraft time tag. The researcher was located in the cargo bay with the switch box, SDA signal analyzer, and computer. The researcher observed the SDA data in real time to determine if the SDA was operating properly. Anomalous SDA performance would be obvious when compared with the ideal reference data collected with the same accelerometer in a laboratory setting.

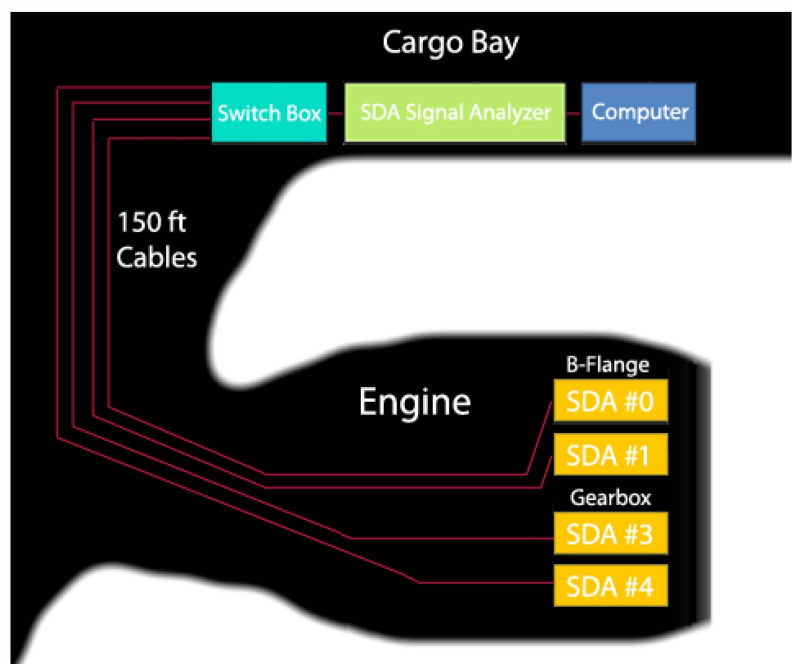

Figure 7. A model of the SDA system and connections installed on the C-17.

One benefit of testing in an actual aircraft is that we have a better understanding of how the cables and accelerometer system interact with the aircraft environment, especially with regard to noise. Noise is introduced in the system via electromagnetic interference from surrounding aircraft systems. Also, vibration of the cables themselves introduces noise. Several techniques were tried to reduce the noise. The cables were isolated with metal sleeves and secured with zip ties. Noise reducing algorithms and averaging also helped. The results suggest that the noise mitigation techniques during engine testing of the SDA were successful.

\section{RESULTS}

The results from VIPR testing of four SDAs are shown in Figs. 8-11. The B-Flange SDA datasets are shown in Figs. 8 and 9. The Gearbox SDA datasets are shown in Figs. 10 and 11. The blue and red plots represent the tight and loose accelerometer attachment conditions, respectively. The lighter color plots, labeled as raw, are the unprocessed cumulative plots of approximately 10 datasets from the named tight or loose attachment condition. To better visualize the noise in the raw datasets, the average of the raw datasets is plotted as a darker color plot and labeled as average. The average is calculated by averaging the 10 datasets and then smoothing using boxcar averaging with a width of 5 data points. 


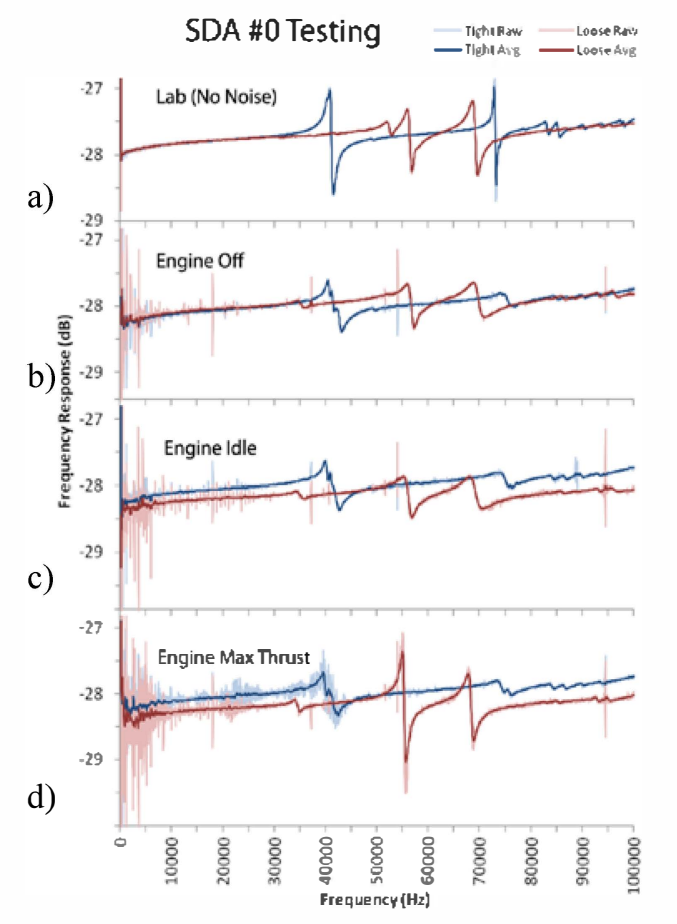

Figure 8. SDA \#0 tight and loose results from the a) laboratory, b) B-Flange engine off, c) B-Flange engine idle, and d) B-Flange engine maximum thrust.

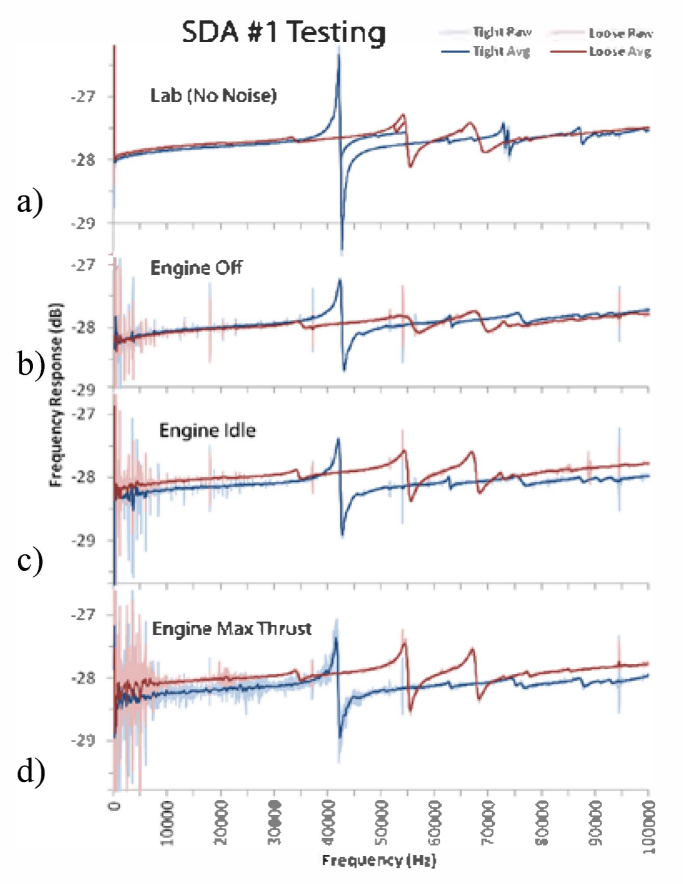

Figure 9. SDA \#1 tight and loose results from the a) laboratory, b) B-Flange engine off, c) B-Flange engine idle, and d) B-Flange engine maximum thrust. a)

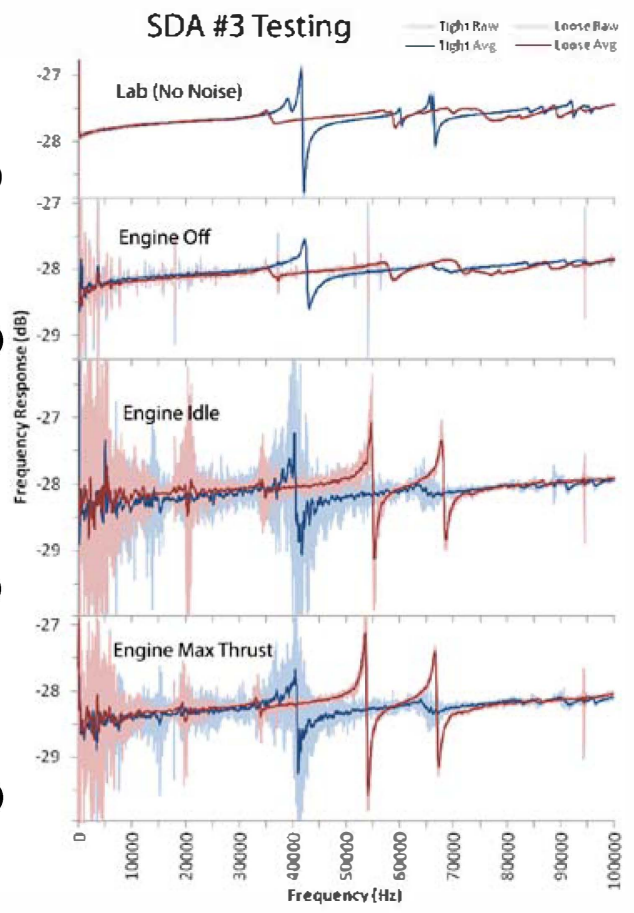

Figure 10. SDA \#3 tight and loose results from the a) laboratory, b) Gearbox engine off, c) Gearbox engine idle, and d) Gearbox engine maximum thrust.

a)

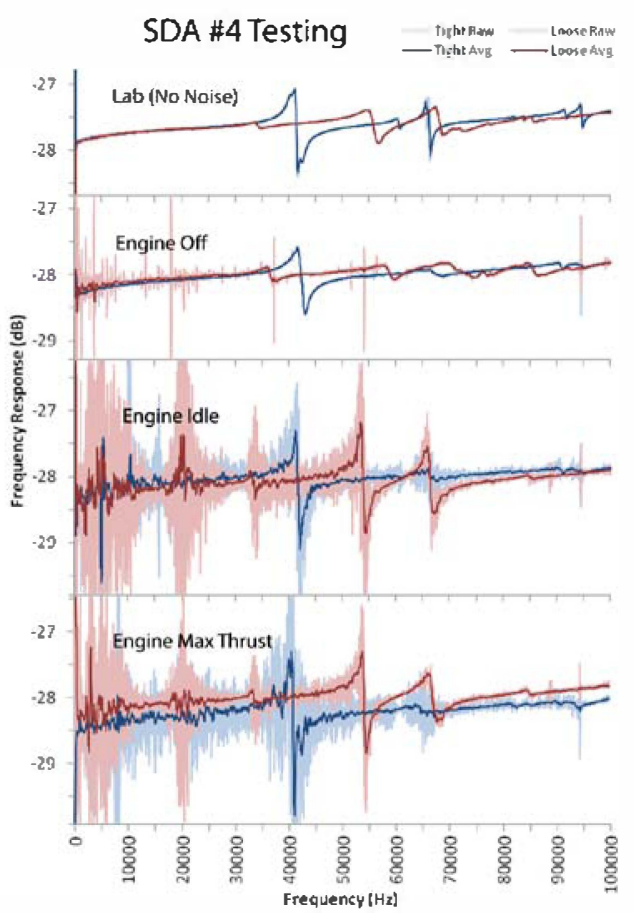

Figure 11. SDA \#4 tight and loose results from the a) laboratory, b) Gearbox engine off, c) Gearbox engine idle, and d) Gearbox engine maximum thrust. 
The laboratory datasets were collected at room temperature, without any vibrations, and with minimal interfering setups (no cable or EMI noise concerns). The mounting hardware and safety wire used in the lab were identical to the configuration used in the $\mathrm{C}-17$ testing. The ideal environment in the lab resulted in stable and consistent SDA signals. For this reason, the laboratory datasets for each SDA were used as the reference signals in the Eqs. 3 and 4 calculation of the Health Index values.

The engine off datasets were taken in the C-17 engine with the engine off during a cool day. The plots show the presence of minor noise in the SDA system. Although the engine was off, vibrations in the low frequency range are present $(<10 \mathrm{kHz})$. The vibrations were most likely caused by the auxiliary power unit located in the lower portion of the aircraft fuselage. The auxiliary power unit was on to provide power to the SDA. Higher frequency noise spikes, most notably seen at frequencies $18 \mathrm{kHz}, 37$ $\mathrm{kHz}, 54 \mathrm{kHz}$, and $95 \mathrm{kHz}$, are present most likely from electrical interference from nearby cables used in other aircraft systems. This electrical interference is also present when the engine is on such as for idle and maximum thrust.

The engine idle datasets were taken in the C-17 engine with the engine at idle. The plots show increased levels of noise present in the SDA system $(<10 \mathrm{kHz})$. Due to the close proximity of the SDA to the engine, engine noise is expected.

The engine maximum thrust datasets were taken in the $\mathrm{C}-17$ engine with the engine at maximum thrust. These plots also show increased noise levels $(<50 \mathrm{kHz})$

For the engine idle and engine maximum thrust conditions the Gearbox location shows a significant increase in noise over the engine off datasets. This broadband noise $(<100 \mathrm{kHz})$ attenuates at the higher frequencies. This noise was not present in the B-Flange datasets.

Vibration measurements were collected from nearby legacy $25 \mathrm{kHz}$ sampling rate B-Flange and $50 \mathrm{kHz}$ sampling rate Gearbox accelerometers. The vibration measurements were taken over 1 minute with the engine off, engine idle, and engine maximum thrust. The Fast Fourier Transforms (FFTs) of this data were calculated for the B-Flange and Gearbox accelerometers and are shown in Figs. 12 and 13 respectively. The FFT vibration results show that the vibrations drop off beyond $5 \mathrm{kHz}$. The acceleration root mean square, $\mathrm{a}_{\text {rms }}$, values are displayed in Table 1. The results show a consistent relationship between the increase in vibrations and the increase in SDA noise except for the Gearbox engine idle and engine maximum thrust data. The large amount of noise present in the data from SDA Gearbox engine idle and maximum thrust levels did not show up in the vibration measurements.

\section{B-Flange Vibration}
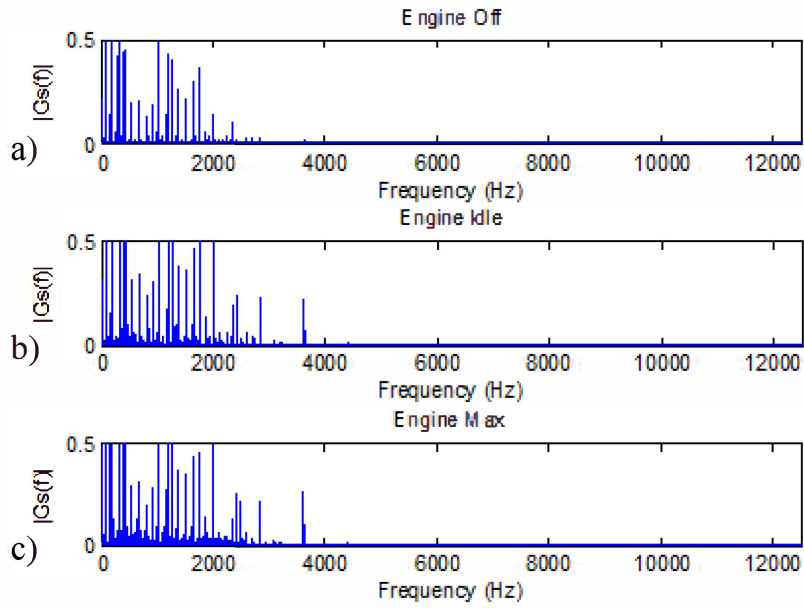

Figure 12. B-Flange legacy accelerometer vibration measurements for 1 minute with the engine a) off, b) idle and c) maximum thrust.

Gearbox Vibration
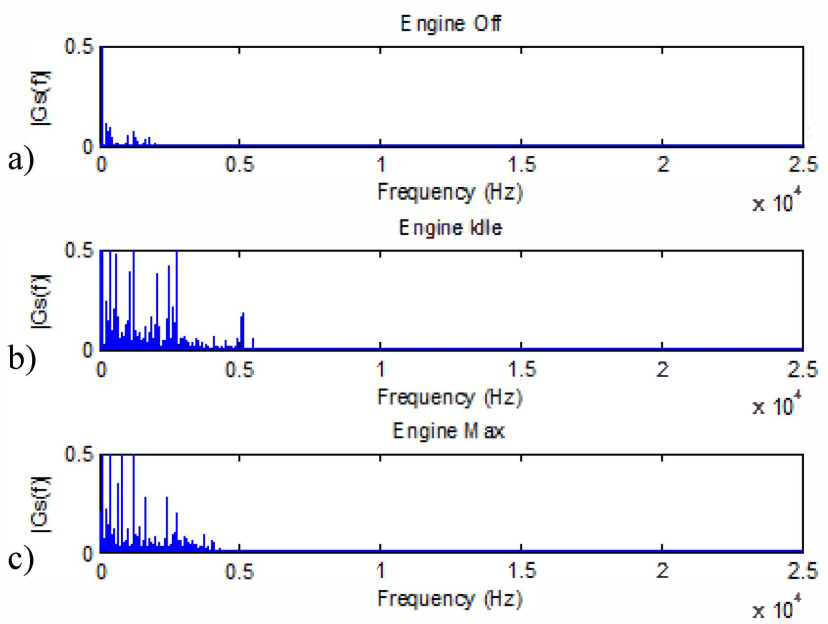

Figure 13. Gearbox legacy accelerometer vibration measurements for 1 minute with the engine a) off, b) idle and c) maximum thrust.

Table 1. B-Flange and Gearbox acceleration measurements for engine off, idle, and maximum thrust.

\begin{tabular}{|c|c|c|}
\hline & B-Flange $\mathrm{a}_{\mathrm{rms}}$ & Gearbox $\mathrm{a}_{\mathrm{rms}}$ \\
\hline Engine Off & 5.73 & 0.62 \\
\hline Engine Idle & 8.83 & 7.00 \\
\hline Engine Max & 9.37 & 5.30 \\
\hline
\end{tabular}


Using the entire collection of raw VIPR data, the Health Index values were calculated using Eqs. 3 and 4 for each dataset. Ten datasets for each condition are averaged together and plotted in Figs. 14 and 15. The average is plotted with 2 standard deviations used as the error bar width.

A tight or loose reference signal for each SDA is cross correlated with the corresponding SDA experimental signals with tight and loose attachment conditions for the laboratory, engine off, engine idle, and engine maximum thrust. Based on results a high correlation was chosen to be any value greater than 0.3 and a low correlation any value less than 0.3. A high value in the tight cross correlation determines the experimental condition to be tightly attached. A low value suggests a fault of some kind. A high value in the loose correlation determines the experimental condition to be loosely attached. A low value suggests otherwise.

The tight reference signal, from the SDA in the laboratory, cross correlated with the experimental signal from the same SDA with the various attachment conditions and environments determines the Health Index values shown in Fig. 14. These results positively identify the tight condition with a high correlation.

\section{Tight Cross Correlation}

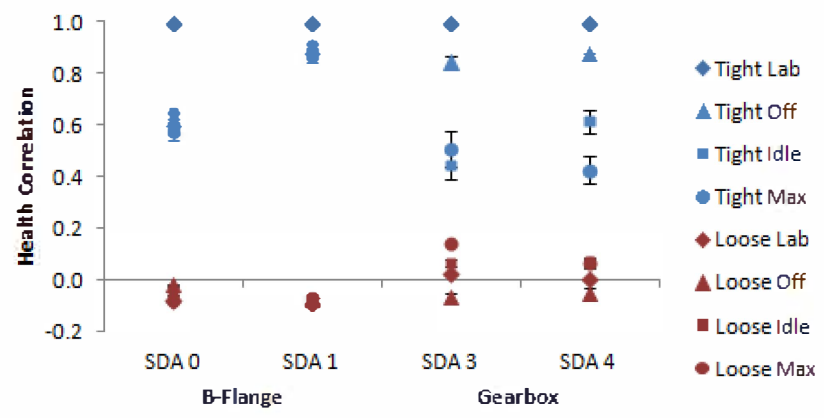

Figure 14. Health Index calculated by using the tight reference correlated with the corresponding SDA for the tight and loose attachment conditions in the laboratory, engine off, idle, and maximum thrust levels.

The loose reference signal, from the SDA in the laboratory, cross correlated with the experimental signal from the same SDA with the various attachment conditions and environments determines the Health Index values shown in Fig. 15. These results positively identify the loose fault with a high correlation except for the SDA 3 experimental loose attachment condition with the engine idle and maximum thrust levels. At these two conditions the Health Index was lower than expected because the reference resonance extrema were not as pronounced as the experimental resonance extrema. This exemplifies the lack of consistency in the loose fault and correspondingly makes it difficult to develop a reliable loose fault reference.

Cross correlating the average datasets improved the correlation when noise was a concern. In the case of the

\section{Loose Cross Correlation}

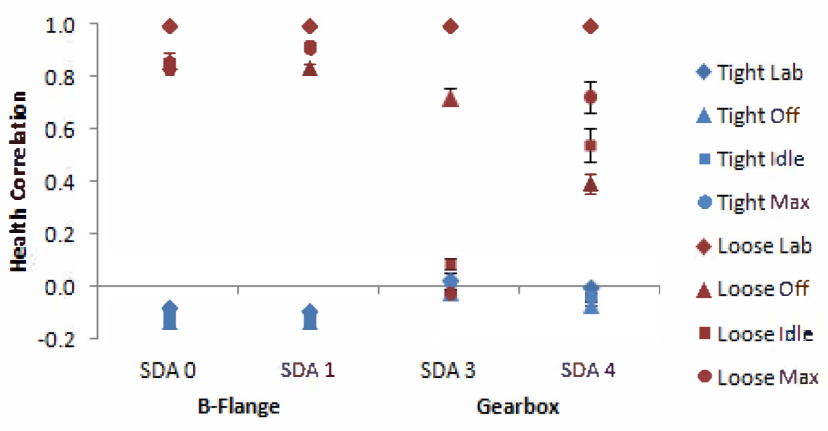

Figure 15. Health Index calculated by the loose reference correlated with the corresponding SDA for the tight and loose attachment conditions in the laboratory, engine off, idle, and maximum thrust levels.

noisy Gearbox datasets for the engine idle and engine maximum thrust, the worst case tight correlation improved from approximately 0.4 to 0.8 . The worst case loose correlation improved from approximately 0.4 to 0.5 . Cross correlating the average data did not improve the lower than expected results for the loose attachment conditions for SDA 3 engine idle and engine max thrust.

\section{Conclusion}

Self diagnostic accelerometers were tested for the first time on a C-17 aircraft engine. The SDAs were installed at the B-Flange and Gearbox engine locations. The engine power levels varied from engine off, engine idle, and engine maximum thrust. The two SDA attachment conditions used were fully tight and loose.

The SDA cross correlation pattern recognition health algorithm successfully discriminates the healthy SDA from the faulty SDA for all tests. The tight reference cross correlated with the tight experimental condition has a minimum correlation of $0.43 \pm 0.05$ which is significantly higher than the maximum correlation of $0.14 \pm 0.01$ for the tight reference cross correlated with the loose experimental condition. If the datasets are first averaged before the cross correlation, the minimum correlation of 0.43 value improves to 0.63 , while the 0.14 value goes to 0.15 . The significant difference in the correlation numbers strengthens the argument that cross correlation pattern recognition can successfully determine whether the SDA is in a healthy tightly attached condition. The SDA system was successful in determining the tightly attached condition even when significant amount of noise was present, as seen in the Gearbox SDA data in the engine idle and maximum thrust levels.

Using the loose cross correlation to confirm the loose fault was successful in all but two test cases: engine idle and max thrust levels for SDA 3. For these two test cases, the resonant frequencies were greater in magnitude than the loose reference SDA 3 in the lab. This exemplifies the lack of consistency in the loose fault. This inconsistency 
makes it difficult to save a reliable loose fault reference for use in the cross correlation pattern method. With the exception of these anomalies the results confirmed the loose fault. The loose reference cross correlated with the loose experimental condition has a minimum correlation of $0.39 \pm 0.04$ which is higher than the maximum correlation of $0.02 \pm 0.00$ for the loose reference cross correlated with the tight experimental condition. If the datasets are first averaged before the cross correlation, the minimum correlation of 0.39 improves to 0.47 , while the 0.02 value goes to 0.04 . The difference in the correlation numbers supports the argument that cross correlation pattern recognition can successfully determine the fault of the SDA. Even so, confirming a specific fault type through cross correlation can be difficult because the resonant frequency pattern in a faulty system may not always be consistent, as it is for the healthy tightly attached condition.

In conclusion, this work is a step towards demonstrating the flight worthiness and robustness of the SDA technology by successfully testing several SDAs in a noisy $\mathrm{C}-17$ engine environment. Future work will include integrating both the SDA diagnostic capability and concurrent vibration sensing from the same accelerometer. Such an upgraded system would demonstrate the combined benefit of an accelerometer able to diagnose its health as well as operate normally as a vibration sensor. Demonstrating that the diagnostic upgrade does not affect normal vibration sensing would then suggest that existing aircraft accelerometers would only stand to benefit by installing the self diagnostic capability.

\section{ACKNOWLEDGMENTS}

This work is supported by the NASA Aviation Safety Program, Vehicle Systems Safety Technologies Project. I'd like to thank Apollo Research Corp. in particular Dave Jaros, Terrence Riggs, and our independent contractor Ken Evans. I'd like to thank all the people that supported VIPR testing at the Dryden Flight Research Center, such as Dave Berger, Mike Venti, Leslie Molzahn, and James Faber, as well as the technical expertise from Michael Delaney, Asif Shahzad, Ray Sadler, Antonio Branco and the SDA and wiring installer, Rich Souza. Lastly, I appreciate the editing by Robert Anderson.

\section{REFERENCES}

[1] M. Davidson and J. Stephens, "Advanced Health Management System for the Space Shuttle Main Engine," in AIAA/ASME/SAE/ASEE Joint Propulsion Conf., Fort Lauderdale, FL, 2004.

[2] D. Simon, et al., "Sensor Needs for Control and Health Management of Intelligent Aircraft Engines," NASA, Cleveland, OH, NASA/TM-2004-213202, 2004.
[3] T. Bickmore, "Real-Time Sensor Data Validation," NASA, Cleveland, OH, NASA CR-195295, 1994.

[4] G.P. Sallee and D.M. Gibbons, "Propulsion System Malfunction Plus Inappropriate Crew Response (PSM+ICR)," AIA, Arlington, VA, AIA/AECMA Project Report, Vol. I, 1998.

[5] S. Clark, et al., "Engine Damage-Related Propulsion System Malfunctions," DOT, Washington, DC, DOT/FAA/AR-08/24, 2008.

[6] W. J. Atherton and P. M. Flanagan, "A Self Diagnostic System for Piezoelectric Sensors," in Proc. 25th AIAA/ASME/SAE/ASEE Joint Propulsion Conf., Monterey, CA, 1989.

[7] P. M. Flanagan and J. Lekki, "A Self-Diagnostic System for the M6 Accelerometer," in Proc. 37th AIAA/ASME/SAE/ASEE Joint Propulsion Conf., Salt Lake City, UT, 2001.

[8] R. Tokars, et al., "Improved signal processing technique leads to more robust Self Diagnostic Accelerometer System," in $48^{\text {th }}$ AIAA Aerospace Science Meeting, Orlando, FL, 2010.

[9] IEEE Standard on Piezoelectricity. ANSI/IEEE Standard 176-1987, 1988.

\section{BIOGRAPHIES}

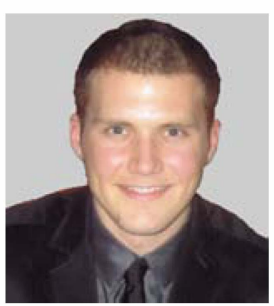

Roger Tokars received a B.S. in Electrical Engineering from Purdue University, West Lafayette in 2007. He has been with NASA $G R C$ for more than 8 years. He has been the study lead for the SDA in the Optical Instrumentation and NDE Branch. He has also been involved in hyperspectral imaging and flow visualizations. Currently he is pursuing his M.S. in Physics from Cleveland State University.

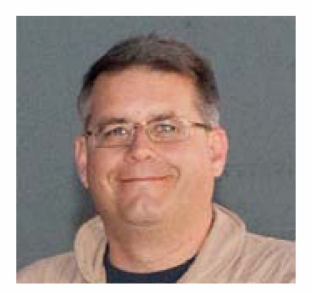

John Lekki received a B.S. in Electrical Engineering from Michigan State University in 1993, M.S. in Physics from Cleveland State University in 2002, and Ph.D in Electrical Engineering from Michigan State University in 2008. He has been with NASA for more than 20 years. He has been the Principal Investigator for the following research activities: Engine Health Management Sensor Technologies, VIPR, Hyperspectral Remote Sensing of freshwater resources, and Quantum Sensing and Communications. He currently works in the Optical Instrumentation and NDE Branch. 